\title{
Design of an Acceleration Gap for Brightness Enhancement in a Reactor-Based Slow Positron Beamline
}

\author{
Y. KuzuYA ${ }^{a, *}$, N. Oshima ${ }^{b}$, A. Kinomura ${ }^{a}$ And A. YabuUchi ${ }^{a}$ \\ ${ }^{a}$ Research Reactor Institute, Kyoto University, Kumatori-cho, Osaka 590-0494, Japan \\ ${ }^{b}$ National Metrology Institute of Japan (NMIJ), \\ National Institute of Advanced Industrial Science and Technology (AIST), Tsukuba-shi, Ibaraki 305-8568, Japan
}

\begin{abstract}
Design of an acceleration gap using mesh electrodes of the brightness enhancement system for the slow positron beamline of Kyoto University research reactor was studied to improve the performance of brightness enhancement. The transmittance and the increase in the angular divergence of the beam resulting from acceleration with the mesh electrode were estimated by trajectory simulations. The effect of the increase in the beam emittance on the beam radius at the focus point was estimated based on the analytical solution of the beam envelope equation. Using the obtained beam transmittance and beam radius, the beam brightness after remoderation was evaluated. Then, the influence of the mesh electrode configuration on the brightness was investigated.
\end{abstract}

DOI: 10.12693/APhysPolA.132.1620

PACS/topics: 78.70.Bj, 41.75.Fr, 29.27.Eg, 41.85.Ja

\section{Introduction}

A slow positron beamline is currently under development for the Kyoto University research Reactor (KUR) [1-4]. The KUR slow positron beamline is composed of a positron source, a brightness enhancement system, a pulsing system, and a measurement chamber. Positrons are generated by pair production from highenergy gamma rays. A W convertor $/ \mathrm{W}$ moderator assembly is used to generate a slow positron beam.

At the KUR, the initial beam diameter is expected to be around $30 \mathrm{~mm}$, which is significantly larger than the size of a typical sample (around $10 \mathrm{~mm}$ ). Therefore, it is desirable to reduce the initial beam size to below $10 \mathrm{~mm}$ while maintaining the beam intensity as high as possible. Therefore, the brightness enhancement system [3] was installed in the beamline.

The brightness enhancement system consists of an acceleration gap, a magnetic lens, a transmission-type re-

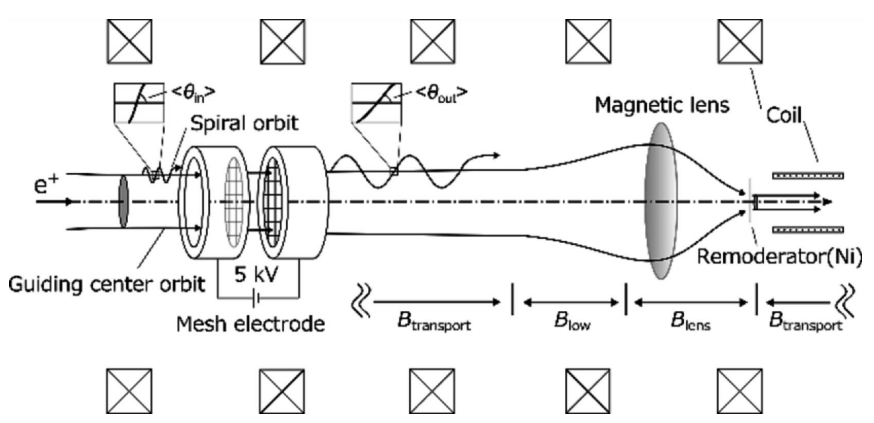

Fig. 1. Schematic view of the brightness enhancement system.

\footnotetext{
* corresponding author; e-mail:

kuzuya.yoshihiro.52e@st.kyoto-u.ac.jp
}

moderator, and several coils (Fig. 1). A positron beam is magnetically guided from the positron source and is subsequently accelerated to $5 \mathrm{keV}$ by the acceleration gap; it then reaches a low magnetic field region $\left(B_{\text {low }}\right.$ in Fig. 1), in which the beam diameter is expanded. The beam is focused onto the remoderator by the magnetic lens, and a fraction of positrons is re-emitted from the opposite surface of the remoderator. In this process, although the total positron flux decreases, the flux density increases, resulting in enhanced brightness. We use a $150-\mathrm{nm}$-thick $\mathrm{Ni}(100)$ thin film [5] as a remoderator purchased from the University of Aarhus. The principle of the brightness enhancement method is described in detail elsewhere $[3,6-9]$.

For brightness enhancement, the acceleration of the positron beam is essential to obtain sufficient focusing through the magnetic lens and is also essential to maximize the re-emission efficiency at the remoderator. In this study, an acceleration gap consisting of a pair of electrodes with two wire meshes was used (Fig. 1). This was referred to as a mesh electrode. The beam is accelerated by the electric field formed between two electrodes. An ideal mesh electrode is virtual mesh with $100 \%$ transmission in which the ratio between the wire diameter $d$ and the aperture width $w$ and the ratio between $w$ to the distance between two electrodes $L$ are negligibly small. Using an ideal mesh electrode, a positron beam can be accelerated through a uniform electric field without any loss in the beam intensity (i.e. the beam transmittance $\left.\eta_{a} \approx 1\right)$. That is, there is no degradation of the beam brightness through acceleration. However, this is not the case with actual mesh electrodes. First, there is a loss of intensity at the wire mesh due to the collision $(d / w>0)$. Secondly, the beam is disturbed by the lens effect due to the distortion of the electric field around the apertures of the wire mesh $(w / L>0)$. The distribution of the angle $\theta_{k}$ along the beam axis of each particle $k$ spreads and the Larmor radius increases, resulting in an increase in 
the angular divergence of the beam $\theta$ (or the beam emittance). Therefore, the beam radius at the focus point $r_{F}$ becomes larger. These two factors cause the degradation of the beam brightness. Thus, the properties of the mesh electrode which affects $\eta_{a}$ and $\theta$ should be optimized.

In this paper, the influence of the parameters of the mesh electrode on $\eta_{a}$ and $\theta$ were estimated by beam trajectory simulations. The effect of the increase in the beam emittance on $r_{F}$ was estimated by an analytical solution of the beam envelop equation. Using the obtained values of $\eta_{a}$ and $r_{F}$, the beam brightness $\beta_{r}$ after the remoderation were estimated. Then, three different mesh electrodes were evaluated to investigate the optimal mesh electrode.

\section{Methods and results}

To understand the dependence of $\eta_{a}$ and $\theta$ on the characteristics of the mesh electrode, three types of wire meshes were investigated. These wire meshes are characterized by following parameters: the number of apertures per unit inch $N$, the aperture width $w$, the wire diameter $d$, and the normalized open screening area $A_{0}$ $\left(=w^{2} /(w+d)^{2}\right)$ (Table I). We chose commercially available wire meshes of $N=30,100$ and 150 apertures/inch. Three commercially available wire meshes of $N=30,100$ or 150 apertures/inch with $d=0.03 \mathrm{~mm}$ were tested in this study, and the distance between two electrodes was fixed to $10 \mathrm{~mm}$; thus, $d / w$ and $w / L$ values were listed in Table I.

TABLE I

Parameters of the mesh electrodes: the number of apertures $N[1 / \mathrm{inch}]$, aperture width $w[\mathrm{~mm}]$, wire diameter $d[\mathrm{~mm}]$, Normalized open screening area $A_{o}, d / w$, and $w / L$

\begin{tabular}{c|c|c|c|c|c}
\hline \hline$N[1 / \mathrm{inch}]$ & $w[\mathrm{~mm}]$ & $d[\mathrm{~mm}]$ & $A_{o}$ & $d / w$ & $w / L$ \\
\hline 30 & 0.82 & 0.030 & 0.93 & 0.037 & 0.082 \\
100 & 0.22 & 0.030 & 0.78 & 0.134 & 0.022 \\
150 & 0.14 & 0.030 & 0.68 & 0.215 & 0.014
\end{tabular}

Generally, the brightness $\beta$ of a positron beam is defined as $[6,8,9]$ :

$$
\beta=I\left(r^{2} \theta^{2} E\right)^{-1} \text {. }
$$

where $I$ is the beam intensity, $r$ is the beam radius, $\theta$ is the angular divergence of the beam, and $E$ is the beam energy. The brightness after remoderation $\beta_{r}$ can be calculated as $\eta_{a} Y_{r} I_{\text {in }}\left(r_{F}^{2} \theta_{r}^{2} E_{r}\right)^{-1}$, where $I_{\text {in }}$ is the beam intensity before acceleration, $Y_{r}$ is the re-emission efficiency of the remoderator, and $\theta_{r}$ and $E_{r}$ are the angular divergence of the beam and the beam energy after remoderation, respectively. These four parameters do not depend on the mesh electrode, so the value of $\beta_{r}$ normalized to the brightness obtained with an ideal mesh electrode is a function of $\eta_{a} r_{F}^{-2}$. $\eta_{a}$ can be calculated as the square of the transmittance, $A_{o}^{2}$ (solid line in Fig. 2a), for the set of the first and second wire meshes.

As the analytical estimation for the increase in $\theta$ may be difficult, beam trajectory simulations were performed using the SIMION code (version 8.1). The results
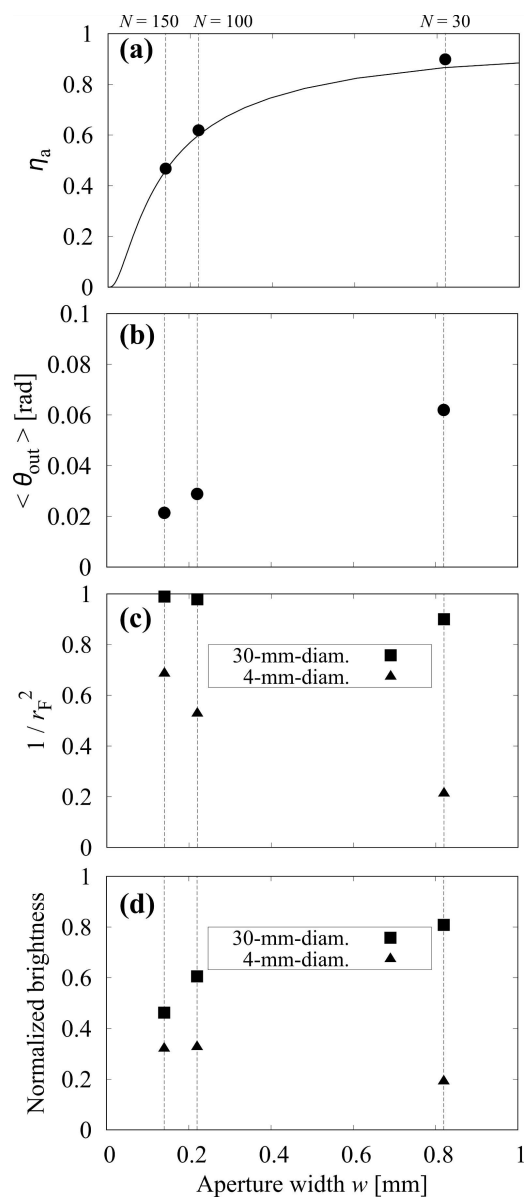

Fig. 2. Results of brightness calculations for various parameters as fuctions of the aperture width $w$ : (a) Theoretically estimated (solid line) and simulated (closed circles) transmitance; (b) Root mean square of the angle of each particle with respect to the beam axis after acceleration; (c) Inverse of the square of the beam radius at the focus point; (d) Normalized brightness after remoderation. Those values are normalized to the value obtained with the ideal mesh electrode.

of the trajectory simulations are shown as $X Y$ crosssectional views in Fig. 3. The simulation volume was $50 \mathrm{~mm} \times 11 \mathrm{~mm} \times 11 \mathrm{~mm}$ on the right-handed Cartesian coordinates ( $X Y Z$ coordinates). The origin corresponds to the center of the beam duct at the left end (see Fig. 3). The beam propagation direction corresponds to the $X$ axis, and the start point of the beam is $X=0 \mathrm{~mm}$. The first and second wire meshes are located at $X=20 \mathrm{~mm}$ and $X=30 \mathrm{~mm}$, respectively. An acceleration voltage of $-5 \mathrm{kV}$ was applied to the second electrode, and the boundary condition between two electrodes along the wall of the beam duct was the natural boundary condition (i.e. the electric field in a normal direction was assumed to zero). The equipotential surfaces around the first wire mesh $(X=18-20 \mathrm{~mm})$ in each case are shown in Fig. 4. It should be noted that these equipotential surfaces were calculated results around the center (i.e. far from the wall) of the beam ducts. In other words, 

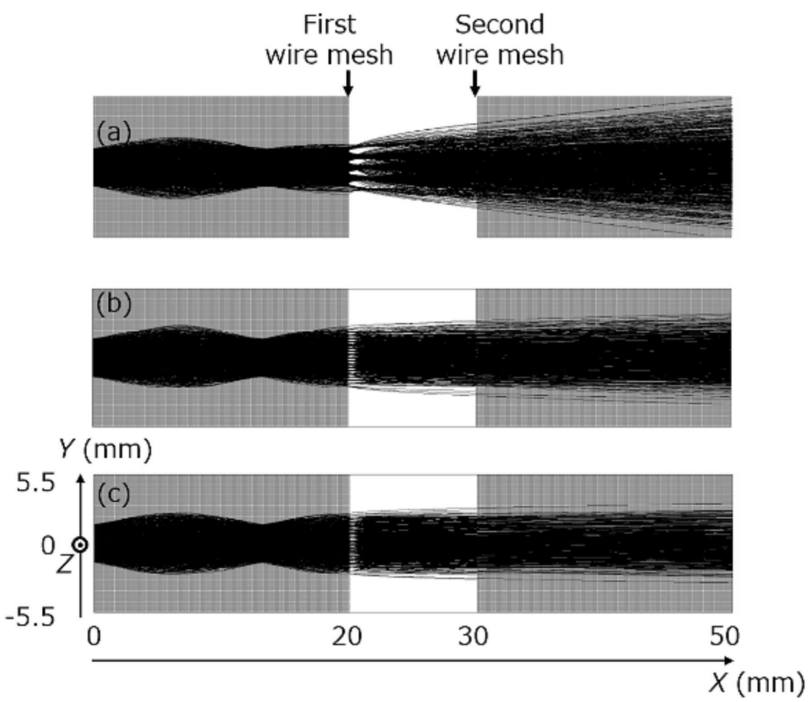

Fig. 3. Results of trajectory simulations with (a) 30 apertures/inch, (b) 100 apertures/inch, and (c) 150 apertures/inch.

equipotential surface patterns near the wall of the beam ducts are different from those that were calculated as shown in Fig. 4. In this study, in order to investigate the dependence of the beam divergence on the mesh structure, the beam size set in the simulation must be much less than the simulated duct size $(11 \mathrm{~mm})$. In addition, if the beam size is equal to or less than $w$, the effect of the wire mesh on the beam could not be evaluated because only one aperture would affect the beam. On the basis of these considerations, the positron source was defined as a uniform circular region of $3 \mathrm{~mm}$ in diameter $\left(Y^{2}+Z^{2} \leq 1.5^{2} \mathrm{~mm}^{2}\right)$ located at $X=0 \mathrm{~mm}$. In this case, the beam diameter was sufficiently smaller than the radial size of the electrode $(11 \mathrm{~mm})$ and was sufficiently larger than $w$. Each positron emitted from the source had an energy of $10 \mathrm{eV}$ and had a random direction in the range of $0-0.305 \mathrm{rad}\left(17.5^{\circ}\right)$ relative to the beam axis, implying that $\theta_{k}$ was uniformly distributed in this range. These parameters were determined based on the known initial beam parameters at the KUR beamline, in which positrons are emitted from $\mathrm{W}$ moderator with re-emission energy of $0-3 \mathrm{eV}[10]$ and are accelerated by an extraction voltage of a few tens $\mathrm{eV}$, giving a $1 / 10$ ratio of transverse energy to longitudinal energy. The number of particles in the simulation $n$ was 10,000. A uniform magnetic field of $B=5 \mathrm{mT}$ along the $X$ axis was applied to the entire simulation domain. In terms of the actual magnetic field around the mesh electrodes, the fluctuation in the magnetic field strength was assumed to be less than $10^{-3}$ to the average value, and the fluctuation in the angle between the magnetic line and the beam axis was assumed to be less than $1 \mathrm{mrad}$. We estimated that these fluctuations were negligibly small in our study, and the uniform magnetic field was reasonable approximation. From the calculation results, we estimated the angular divergence of the beam after acceleration $\theta_{\text {out }}$ according to the root mean square of $\theta_{k}$ using the following equation:

$$
\left\langle\theta_{\text {out }}\right\rangle=\sqrt{\frac{1}{n} \sum_{k=1}^{n} \theta_{k}^{2} .}
$$

The calculated values of $\eta_{a}$ and $\left\langle\theta_{\text {out }}\right\rangle$ are shown in Figure $2 \mathrm{a}$ (closed circles) and $2 \mathrm{~b}$, respectively.

The value of $r_{F}$ was estimated by applying the analytical solution of the beam envelope equation reported by Oshima et al. [7]. Under the assumption that the electromagnetic field along the beamline has a rotational symmetry, there exists an invariant $Q^{2}$ which is a function of the beam parameters. Applying the paraxial approximation, $Q^{2}$ can be described by the following equation:

$$
Q^{2}=V\left\langle\theta^{2}\right\rangle r^{2}+\frac{q}{8 m} B^{2} r^{4},
$$

where $V$ is the beam acceleration voltage, $r$ is the beam radius, $m$ and $q$ are the mass and charge of a positron, respectively, and $B$ is the magnetic field. $r_{F}$ can be determined using the following equation:

$$
r_{F}^{2}=\left(\frac{Q}{\sqrt{V} \alpha}\right)^{2}+\left(\frac{C s \alpha^{3}}{4}\right)^{2},
$$

where $\alpha$ is the convergence angle of the positron beam, and $C s$ is the spherical aberration coefficient of the magnetic lens [11]. The value of $r_{F}$ was estimated using Eqs. (3) and (4). The first term on the right-hand side of Eq. (3) expresses the beam emittance, and its value increases as $\left\langle\theta_{\text {out }}\right\rangle$ increases; the second term does not change during acceleration. Thus, $r_{F}$ was estimated based on $\left\langle\theta_{\text {out }}\right\rangle$ and the parameters of the KUR beam $(r=15 \mathrm{~mm}, B=5 \mathrm{mT}, C s=36 \mathrm{~mm}) . C s$ was estimated numerically by using the paraxial ray equation [11]. The inverse of the square of $r_{F}$ is shown for several values of $w$ in Fig. 2c. For comparison, the same parameters in the case of a beam radius of $2.0 \mathrm{~mm}$, which corresponds to positron beams from radioisotope (RI) sources [12], is also shown.

Using the estimated values of $\eta_{a}$ and $r_{F}$ as described above, the normalized brightness was calculated for several values of $w$ (Fig. $2 \mathrm{~d}$ ).

\section{Discussion}

The results of the trajectory simulations shown in Fig. 3 clearly indicate that the divergence of the beam increases as the aperture width is widened. The distortion of the electric field around each aperture (Fig. 4) works as a lens for the positrons. As the aperture width increases, the distortion of the electric field increases, and hence, the beam diverged more significantly. Around the second wire mesh, similar distortions were also observed (not shown) in the electric field. However, the lens effect of these distortions was less significant because the beam had already been accelerated to $\approx 5 \mathrm{keV}$ upon reaching the second wire mesh, and the influence of a distortion in an electric field becomes relatively small for higherenergy beams. These results suggest more suitable mesh electrodes could be selected: a first mesh with a smaller $w / L$ ratio and a second mesh with a smaller $d / w$ ratio. 


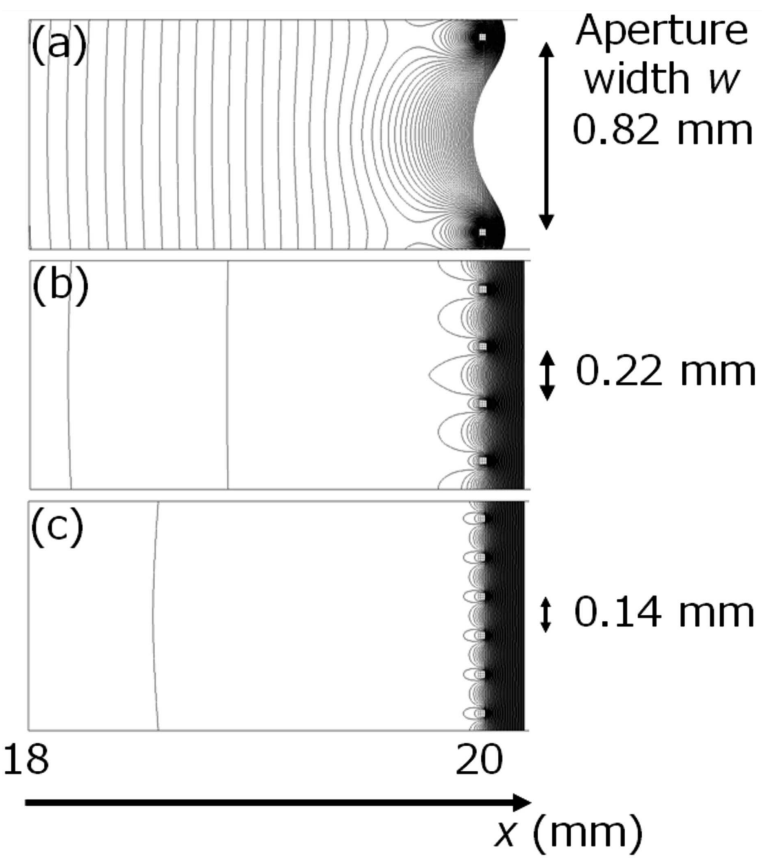

Fig. 4. Equipotential surfaces around the first mesh potentials ranging from -90 to $0 \mathrm{~V}$ with a step of $1 \mathrm{~V}$ for (a) 30 apertures/inch, (b) 100 apertures/inch, and (c) 150 apertures/inch.

Figure $2 \mathrm{a}$ shows the $\eta_{a}$ values estimated based on the values of $A_{o}^{2}$ (solid line) and the corresponding simulated values (closed circles). These results agreed with each other, indicating that the beam transmittance can be estimated without characterizing the distortion of the electric field. The inverse squares of the $r_{F}$ values with $N=$ 30,100 , and 150 normalized to the $r_{F}$ value obtained with the ideal mesh electrodes are shown in Fig. 2c. The values vary slightly with the $30-\mathrm{mm}$-diameter beam, but they differ significantly with the 4-mm-diameter beam. This is because the increase in $\left\langle\theta_{\text {out }}\right\rangle$ affects $Q^{2}$ (i.e. $r_{F}$ ) more significantly in the 4-mm-diameter beam. The evaluated normalized brightness values shown in Fig. 2 d indicate that, with a 30 -mm-diameter beam, the brightness can be maximized using a wire mesh with $N=30$ because the effect of $\eta_{a}$ on the brightness is more significant than the influence of $r_{F}$ on the brightness. The brightness values when $2 r=40,50$, and $60 \mathrm{~mm}$ were also evaluated and the highest brightness values were obtained with a wire mesh of $N=30$. On the contrary, in the case of a 4-mm-diameter beam, wire meshes with $N=100$ or 150 resulted in higher brightness than a wire mesh of $N=30$ because, in this case, the $r_{F}$ value had a more significant effect on the brightness than $\eta_{a}$.

Another potential candidate for the acceleration of a positron beam is an acceleration tube, which consists of several ring electrodes without any wire meshes. It may be informative to make a critical comparison between a mesh electrode and an acceleration tube to further improve the performance of the brightness enhancement system.

\section{Summary}

Design of an acceleration gap with mesh electrodes for the brightness enhancement system of the KUR slow positron beamline was studied to improve the performance of brightness enhancement. The beam transmittance and the increase in the angular divergence of the beam at the mesh electrode were estimated by trajectory simulations. The effect of an increase in the beam emittance on the beam radius at the focus point were estimated by applying an analytical solution to the beam envelope equation. From these estimations, the beam brightness after the remoderation was evaluated. The obtained results suggest that the optimal condition for relatively large-diameter $(>30 \mathrm{~mm})$ beams, such as that of the reactor-based positron source, can be attained using a coarse mesh (i.e. an electrode with a wire mesh of 30 apertures/inch).

\section{Acknowledgments}

We would like to thank Drs. K. Sato (Kagoshima Univ.), Q. Xu (KURRI), and K. Ito (AIST) for valuable discussion and also thank our colleagues at AIST, Tohoku Univ., Kyoto Univ., KEK, and the Tokyo Univ. of Science for their invaluable assistance.

\section{References}

[1] Q. Xu, K. Sato, T. Yoshiie, T. Sano, H. Kawabe, Y. Nagai, K. Nagumo, K. Inoue, T. Toyama, N. Oshima, A. Kinomura, Y. Shirai, J. Phys. Conf. Ser. 505, 012030 (2014).

[2] K. Sato, Q. Xu, T. Yoshiie, T. Sano, H. Kawabe, Y. Nagai, K. Nagumo, K. Inoue, T. Toyama, N. Oshima, A. Kinomura, Y. Shirai, Nucl. Instrum. Methods Phys. Res. B 342, 104 (2015).

[3] Y. Kuzuya, N. Oshima, A. Kinomura, A. Yabuuchi, K. Sato, Q. Xu, J. Phys. Conf. Ser. 791, 012012 (2017).

[4] A. Yabuuchi, A. Kinomura, Y. Kuzuya, K. Sato, Q. Xu, N. Oshima, B.E. O'Rourke, J. Phys. Conf. Ser. 791, 012013 (2017).

[5] P.J. Shultz, E.M. Gullikson, A.P. Mills, Jr., Phys. Rev. B 34, 442 (1986).

[6] A.P. Mills. Jr., Appl. Phys. 23, 189 (1980).

[7] N. Oshima, R. Suzuki, T. Ohdaira, A. Kinomura, T. Narumi, A. Uedono, M. Fujinami, J. Appl. Phys. 103, 094916 (2008).

[8] M. Maekawa, K. Wada, Y. Fukaya, A. Kawasuso, I. Mochizuki, T. Shidara, T. Hyodo, Eur. Phys. J. D 68, 165 (2012).

[9] M. Fujinami, S. Jinno, M. Fukuzumi, T. Kawaguchi, K. Oguma, T. Akahane, Anal. Sci. 24, 73 (2008).

[10] R. Suzuki, G. Amarendra, T. Ohdaira, T. Mikado, Appl. Surf. Sci. 149, 66 (1999).

[11] P.W. Hawkes, E. Kasper, Principles of Electron $O p$ tics, Academic, London 1989.

[12] R. Krause-Rehberg, N. van der Walt, L. Büttner, F. Börner, Nucl. Instrum. Methods Phys. Res. B 221, 165 (2004). 